\title{
sciendo
}

\section{Space Emergence in Contemporary Physics: Why We Do Not Need Fundamentality, Layers of Reality and Emergence}

\begin{abstract}
Baptiste Le Bihan
University of Geneva

DOI: $10.2478 /$ disp-2018-0004

BIBLID [0873-626X (2018) 49; pp.71-95]

Abstract

'Space does not exist fundamentally: it emerges from a more fundamental non-spatial structure.' This intriguing claim appears in various research programs in contemporary physics. Philosophers of physics tend to believe that this claim entails either that spacetime does not exist, or that it is derivatively real. In this article, I introduce and defend a third metaphysical interpretation of the claim: reductionism about space. I argue that, as a result, there is no need to subscribe to fundamentality, layers of reality and emergence in order to analyse the constitution of space by non-spatial entities. It follows that space constitution, if borne out, does not provide empirical evidence in favour of a stratified, Aristotelian in spirit, metaphysics. The view will be described in relation to two particular research programs in contemporary physics: wave function realism and loop quantum gravity.
\end{abstract}

\section{Keywords}

Spacetime, space, emergence, fundamentality, levels of reality.

1 Recovering space and spacetime in contemporary physics

'Space does not exist fundamentally: it emerges somehow from a more fundamental non-spatial structure.' This intriguing claim appears in various approaches to quantum mechanics and quantum gravity. In quantum mechanics, proponents of wave function realism (also called 'wave function monism' or 'configuration space realism') argue that wave functions are genuine entities, physical fields, living in a physical counterpart of the configuration space (that describes all 
the possible state of the physical system under consideration), namely, in a structure made of $3 \mathrm{~N}$ dimensions, $N$ corresponding to the number of physical particles. Therefore, in this account, the actual world is made of a gigantic number of dimensions, and is inhabited by a physically real wave function, itself understood as a collection of properties assigned to coordinates in a space very different from the ordinary space. A problem is then to understand the metaphysical status of the ordinary space we experience on a daily basis, and its relation with the physical configuration space. In quantum gravity, research programs such as loop quantum gravity and string theory state that the relativistic spacetime is not fundamentally real and emerges from a non-spatio-temporal ontology. ${ }^{1}$ Here again, one problem is to understand the metaphysical status of the emerging structure (the relativistic spacetime). The proposal that space or spacetime is not fundamentally real is far more radical that the relationist claim - Leibnizian in spirit - that spatial or spatio-temporal relations depend on their relata, space or spacetime being identified with the collection of these relations. What comes under attack with the phenomenon of space emergence is not the substantiality of space (ordinary space or relativistic spacetime), but the fundamental existence of its structure: if borne out, space emergence would entail that space, with its structural organization - as described by general relativity with the metric field, and our ordinary phenomenologydoes not exist fundamentally (or alternatively, that another space exists fundamentally, but one which differs both from our familiar phenomenological space and from the spacetime of general relativity in the case of quantum gravity).

If one of these approaches turns out to be right, then it seems that we will have a novel reason to accept the existence of levels of reality connected by relations of ontological priority. Indeed, at first glance, the claim that ordinary space (or relativistic spacetime) is not fundamental invites two main readings: either space is not real at all

\footnotetext{
${ }^{1}$ Similar claims appear in most research programs in quantum gravity. For a general and more technical review of the features usually ascribed to space and time and said to be missing, see Huggett and Wüthrich 2013 and Le Bihan and Linnemann (forthcoming). For a discussion of the issue in string theory, cf. Huggett 2017. For a defense of the view that quantum gravity does not necessarily entail that spacetime is not fundamental, see Yates 2018.
} 
or it is non-fundamentally real. In other words, the first interpretation amounts to the view that space is emergent (or does not exist fundamentally) because space does not exist simpliciter, suggesting that emergence should be explained away as a form of illusion. ${ }^{2}$ This eliminativist view leads to the disturbing consequence that almost everything we take to be true about space is literally false. According to the second approach, the derivative space view, space is emergent because space does exist derivatively. It suggests a layered picture of the natural world with at least two levels of reality, the space level being less fundamental than the non-spatial fundamental structure, this more-fundamental-than relation being here identified with a relation of ontological priority. Although eliminativism has been voiced in the philosophy of physics literature, most philosophers of physics are attracted by this second interpretation and take the disappearance of space or spacetime to engage the existence of at least two levels of reality: a more fundamental non-spatio-temporal level and a less fundamental spatio-temporal level.

As a result, space emergence seems, prima facie, to entail the existence of some levels of reality. ${ }^{3}$ The idea that reality is layered in ontological levels is not new. Importantly, we find it in discussions on the status of special sciences. One possible interpretation of the non-reductionist claim that special sciences such as biology or cognitive psychology do not reduce, semantically, to fundamental physics is that the entities engaged by these special sciences may not be identified with the entities posited by fundamental physics. If true, then the natural world is layered in several levels of reality, one

${ }^{2}$ Emergence would then be epistemic with no counterpart existing in the world. Of course, understanding exactly how this is possible is part of the challenge the eliminativist has to meet.

${ }^{3}$ Depending on what we mean by the expression 'level of reality', one might argue that a world made of fundamental and non-fundamental entities should not be understood as a world in which there are levels of reality. It should be clear, however, that in the case of space emergence, non-fundamental spatial entities are taken to be all located in the same domain of description, while fundamental entities all are located in another domain of description, in such a way that if we want to claim that these domains of description represent genuine distinct ontological domains, the talk of levels of reality is perfectly justified. 
corresponding to each of the special sciences. ${ }^{4}$ As such, a proponent of levels in special sciences might argue that space emergence would provide us with new evidence that the natural world is layered. Could it be that even within physics we might find evidence of levels and that anti-reductionism is the best approach to special sciences?

I will suggest otherwise and argue that space emergence does not require positing ontological levels, relations of ontological priority, or emergence within physics. In order to argue for this claim, I will introduce and motivate the mereological bundle theory of space: $:^{5}$ what we call a 'derivative' or a 'non-fundamental' space is in fact a mereological bundle of proper parts of the 'maximal structure', namely the whole cosmos, whatever its exact (non-spatial) nature turns out to be.

At this stage, a word of caution is in order. This essay aims at prompting a discussion between metaphysicians about how we should best understand philosophically what has been called by physicists working in quantum gravity 'space emergence'. The topic is especially difficult since it lies at the intersection of various fields: analytic metaphysics, philosophy of quantum gravity and, it has been suggested, philosophy of emergence. Note, however, that the expression 'emergence' should be understood in this context as a neutral expression, a placeholder for a problem, which does not commit one to any particular interpretation of the nature of the relation. This point deserves our attention since the term has a different meaning in philosophy and general philosophy of science on the one hand, and in philosophy of physics and physics on the other hand - the separation may be drawn differently, but what matters here is that there exist two different terminological traditions. In the field of general philosophy, the relation of emergence is a very specific notion associated with highly specific features: especially, emergent entities, properties or powers are regarded as not owned by the system from which they emerge, against reductionism (the view originates in the tradition of

\footnotetext{
${ }^{4}$ The layered approach is famously criticised by John Heil (2003a, 2003b).

${ }^{5}$ More precisely, I will defend that the mereological approach is a possible abstract characterization of space emergence. This possibility is enough to show that space emergence does not entail the existence of ontological levels.
} 
British emergentism ${ }^{6}$ and has been recently discussed by several philosophers, cf. Humphreys 2016 for a review). In contrast, in physics and philosophy of physics proper, the relation is generally regarded as a generic one which still has to be interpreted further and is even consistent with reductionism (see Butterfield 2011, Crowther 2018).

The fact that physicists and philosophers of physics have referred to the problem of spacetime emergence by using the word 'emergence' is a bit unfortunate when one wishes to further analyse the notion: indeed, it already suggests that the relation obtaining between the non-spatio-temporal and the spatio-temporal is a relation of (philosophical) emergence, a claim that is not made by most philosophers of quantum gravity. The two notions of emergence share a very different story. In physics, the expression signals an issue when in philosophy it corresponds to a highly specific notion, quite at odds with the reductionist motivations that we find in the philosophy of quantum gravity community.

As a solution, I propose to rename the problem as a problem of spacetime constitution, in reference to the problem of material constitution in the tradition of analytic metaphysics. This problem begins by examining the relation obtaining between material objects such as statues and the matter they are made of. In a nutshell, the two objects seems to be numerically identical because they share the same volume of spacetime; but they seem different since they have different modal properties: for instance, the statue may not survive a reshape, contrary to the lump of clay it is made of, entailing that the statue and the lump of clay instantiate different modal properties. In both contexts (the problems of material constitution and spacetime constitution), we may then formulate the view that constitution is emergence, implying that some genuinely new properties pop into reality at the derivative level. Although, for the sake of brevity, I will not develop in detail the comparison here, the moral to be drawn is that constitution may be regarded as a more neutral relation and that, the use of the expression 'space emergence' by physicists does not entail that the philosophical notion of emergence should play a role in this context.

${ }^{6}$ See McLaughlin 1992, Thomas 2013.

${ }^{7}$ But see e.g. Ladyman and Ross 2007 for a non-reductionist and naturalist interpretation of science. 
In the next section of the paper, I will introduce briefly two potential cases of space constitution: wave function realism and loop quantum gravity. In sections three and four, I will critically discuss, respectively, the eliminativist view and the derivative space view in order to motivate reductionism as a third promising alternative. In sections five and six, I will introduce mereology and logical mereology, arguing that space constitution can be analysed in terms of logical composition, avoiding any reference to ontological levels.

\section{Wave function realism and loop quantum gravity}

In this section, I illustrate the idea of space constitution by describing two particular cases, one which is a possible interpretation (or theory) of quantum mechanics, the other a research program in quantum gravity: loop quantum gravity. But many other examples of space emergence may be found in the quantum gravity literature, and the general ontological interpretation that I will propose, by its abstract nature, should apply to many, if not all, of them. ${ }^{8}$ LQG is one program among many in quantum gravity. These programs aim at finding an explanation of quantum gravity, either by starting with a GR framework, then introducing quantum aspects in the account (as with LQG), or in trying to unify general relativity with the standard model of particle physics by building a new theory (as with string theory). ${ }^{9}$ None of these research programs are empirically confirmed, though. However, we may hope that at least one of these

${ }^{8}$ In string theory, the situation is not clear yet. What seems clear, though, is that if relativistic spacetime is real, it is numerically distinct from the space in which the strings live (see Huggett 2017) because of the phenomenon of duality (see e.g. Le Bihan and Read forthcoming). For a general review of spacetime constitution in quantum gravity, see Huggett and Wüthrich 2013 and Le Bihan and Linnemann forthcoming.

${ }^{9}$ Right now, our two most fundamental physical theories are general relativity and the standard model of particle physics (leaving aside the possibility that macroscopic thermodynamics is also fundamental, see Ladyman and Ross 2007). The two theories describe different physics and are in tension to explain phenomena where gravitational effects (the playground of GR) meet quantum effects (an aspect of quantum mechanics, also covered by the standard model of particle physics), namely phenomena such as black holes and the very early universe. 
research programs is on the right track and it is interesting to look at the features shared by most of these approaches. Space constitution is one such a shared feature. In brief, space emergence is not specific to LQG but to most of the research programs in quantum gravityin such a way that LQG is representative of the kind of scientific revolution that is to be expected from physics. So in what follows, LQG should be taken as a particular example of the situation we encounter in quantum gravity with respect to space constitution.

According to the Wave Function Realist interpretation of quantum mechanics (WFR hereafter), our ordinary 3D space is constituted by a more fundamental space of $3 N$ dimensions, $N$ being the number of fundamental physical particles ${ }^{10}$ in the universe, or more accurately, the number of apparent fundamental physical particles: properly speaking, fundamental physical particles do not exist (see e.g. Monton 2002, 2006; Lewis 2004 and Ney 2012, 2015). In this approach, the wave function is not (merely) a mathematical tool used to describe properties of a particular physical system: the wave function is a genuine entity living in an exotic ${ }^{11}$ space (distinct from the ordinary space). An interesting aspect of WFR is that it explains non-local Bell correlations, the fact that some physical values of proper parts of dispersed physical systems are anti-correlated. In WFR, these anti-correlations are explained by a simple fact: two apparent numerically distinct entities $x$ and $y$ in 3D space share the same location in a higher-dimensional space, as aspects of the wave function. Of course, the $3 N$-dimensional space ( $3 N$ space from now on) is just one possible ontological interpretation (or one possible theory) of quantum mechanics, but other interpretations will not be discussed in this article. ${ }^{12}$

Take note that in WFR, the problem is only about space and not

10 'Fundamental particles' refer here to 'mereological simples'.

${ }^{11}$ One may argue that the configuration space is not that exotic since physicists use them all the time. However, what is exotic is the physical configuration space understood as a physical structure lying behind the mathematical configuration space.

${ }^{12}$ Take note that wave function realism is an interpretation consistent with several other classical interpretations of quantum mechanics, such as the Bohmian and the Many-Worlds interpretations. 
about time. ${ }^{13}$ The wave function lives in a $3 \mathrm{~N}$ space where time flows or, in the framework of a B-theory of time, ${ }^{14}$ where there is a further time dimension. This time dimension is regarded as being identical to macroscopic time (namely, time as we ordinarily conceive of it). There, the problem is to understand how fundamental space gives rise to a particular derivative space, being granted that fundamental time is identical with derivative time - in such a way that it is more reasonable to just refer to time since the fundamental/derivative distinction does not apply to time in this context.

Let us now examine the ontological picture of loop quantum gravity in order to see how it relates to the situation of WFR. According to Loop Quantum Gravity (LQG hereafter), spacetime is not fundamentally real: what there is instead are entities described by 'spin networks', namely collections of nodes and relations (the loops) between these nodes (see Rovelli 2004 and Rovelli and Vidotto 2014; for a summary aimed at philosophers cf. Huggett and Wüthrich 2013). When we apply dynamics to 3D spin networks, we obtain a 4D system called 'spin foam'. In LQG, the spin foam is taken to be sufficiently similar to GR spacetime to explain the empirical success of general relativity. These spin networks represent discretely valued volumes and areas, generically in superpositions, prompting a question about the relation between these discrete quantum structures and the continuous classical GR structure. Furthermore, the organization of the LQG structure does not always correspond to the structure of the GR structure: some relations of adjacency in the LQG structure correspond to relations of large distances in the GR structure (see Huggett and Wüthrich, 2013). In short, an approximation of GR spacetime emerges 'somehow' from a more fundamental structure made of entities that are discrete and in a state of disordered locality. ${ }^{15}$

${ }^{13}$ Arguably, the discussion aims at explaining the connection between the $3 \mathrm{~N}$-world and ordinary space and time. However, it is worth asking how it could be, were such a picture to be true, that GR physics is predictively successful. So not only has the proponent of WFR to offer a story about the connection between the fundamental $3 \mathrm{~N}$-world and phenomenal space and time, but also an explanation of the predictive success of GR, which posits a four-dimensional structure.

${ }^{14}$ See e.g. Oaklander 1987 and Mellor 1998.

${ }^{15}$ A specific issue is the relation between the quantum LQG structure and the 
So let us assume that spin foam is the fundamental structure and GR spacetime is the relevant derivative structure. We may distinguish between two conceptual issues with the disappearance of spacetime in LQG: how are we to construe the emergence of something close enough to the world described by general relativity in order to explain its predictive success (problem of GR success)? And how are we going to make sense of the possibility of measurement somewhere and somewhen? Can we appeal to measurements occurring in space and time to justify the claim that space and time do not exist (problem of empirical (in)coherence) ${ }^{16}$ The two problems of GR success and empirical coherence rest on the very same issue: what is the metaphysical status of this constituted spacetime? And why is it that this particular ordinary space exists rather than another space consistent with the fundamental theory? As we shall see, in order to solve these issues, philosophers of physics usually posit the existence of a derivative space, both in LQG and in WFR, thereby entailing the existence of levels of reality within physics.

\section{No space?}

According to eliminativism, space is not fundamentally real because it does not exist tout court. This is probably the more straightforward way to reject the idea that space constitution entails the existence of levels of reality. 'Constitution' would be a misnomer or, at best, constitution in a narrow epistemic sense: due to some features of our conceptual and perceptual apparatus, space seems to exist. But there is no genuine, mind-independent, constitution of space since there is no space. In this interpretation, the phenomenology of space and time relates directly to the fundamental non-spatial ontology, without positing an in-between physical derivative spatial structure. For instance, if we follow Albert (2015: 128-9), in the background

non-quantum GR structure. But since the measurement problem is a specific issue, already to be found in quantum mechanics and quantum field theory, it is not necessarily related to the problem of space constitution and I will leave it aside. It could be that the measurement problem must play a role in solving the problem of spacetime constitution but I will not purse this line of thought in this essay.

${ }^{16}$ See Wüthrich 2017. 
of WFR, 3D objects can be picked through functional realization. ${ }^{17}$ This functional role is merely a formal possibility to build objects though, and there are no 3D objects obtaining in the world. Unlike the derivative space view, eliminativism does not require postulating a stratified ontology with distinct levels of reality. And it does not require positing a special relation connecting the entities inhabiting the distinct levels. So eliminativism is less demanding than the derivative space view and avoids any commitment to levels of reality.

However, this interpretation does not come for free. In the metaphysical and phenomenological literature, it is quite common to defend the view that time does not flow, and that the notion of flow corresponds to a perceptual artefact (see for instance Paul 2010 and Benovsky 2015). Most metaphysicians of time agree on the existence of temporal relations. Following the classical distinction between A-properties (the properties of being past, present, future, or being two days past or future for instance) and B- or C-relations, ${ }^{18}$ the view is that temporal relations are mind-independent components of the world. Eliminativism is far more radical since it denies the reality of spatial, temporal and spatio-temporal relations. Eliminativists have to deny that the very basic features common to time and space (like metrical and topological aspects) are real. So the challenge the eliminativist has to address is really radical. It is far from obvious that it will be possible to come up with a consistent story about phenomenal time and space as perceptual artefacts in a background ontology in which there are no spatial or temporal relations. Perhaps such a story could be offered, but for now it is not clear what it would look like, and so, we should not take for granted that there is room for such an explanation. As we shall see, the notion of derivative space avoids many of these issues but generates new ones.

${ }^{17}$ Lam and Wüthrich (forthcoming) have proposed to identify spacetime emergence with spacetime realization, but their view is not tied to an eliminativist understanding and remains consistent with a derivative view.

${ }^{18}$ B-relations are temporal relations of order (intrinsically orientated), Crelations are non-temporal relations of order (not intrinsically orientated). In McTaggart's picture (1908), the flow of time (the transition of A-properties) in the $\mathrm{C}$-dimension made of $\mathrm{C}$-relations, generates an orientation of the dimension and results in B-relations. In the tradition originating in Russell, B-relations are primitive notions (the relations are primitively orientated) and C-relations and Aproperties are not real. See for instance Oaklander (1987) and Le Bihan (2015). 


\section{Derivative space}

According to the derivative space view, space is not fundamentally real because it is only derivatively real (the view has been voiced for instance by Wüthrich 2017: 298, cf. Le Bihan 2018 for further discussion). Space is grounded in, or is built from, a more fundamental ontology. There are many possible ways to think of the connecting device obtaining between the two layers and grounding or building the upper entities. One may construe the relation as a grounding relation. Note, however, that this relation has to be ontological - it cannot be merely a mathematical procedure (as with Albert's notion of functional realization that suggests an eliminativist picture).

The derivative view suggests not only that the world is stratified, but also that the layers are related by a connecting relation and that the derivative structure is less fundamental than the fundamental structure. Take note that there is not necessarily a connection between the stratified view and the existence of fundamentality relations. We may well discover one day that the world is stratified although no relation of fundamentality is connecting the layers. Also, there is no logical connection between the existence of fundamentality relations and the view that this or that particular level is more fundamental than the others. Physics seems to construe the small-scale world as being more fundamental than the macroscopic and the cosmological scales. Still, it might be that the more fundamental level is the macroscopic level or the cosmological level (see Schaffer 2010).

By positing a derivative space, a physical creature both distinct from the fundamental structure and the phenomenal space and time, one lays the groundwork for an answer to the phenomenal worry. If we perceive space and time, this is simply because space and time are real, although derivatively real. We have direct phenomenal access to these derivative entities and this is why our daily life takes place in a spatial and temporal environment. Also, the derivative view explains the empirical success of GR: a derivative structure, close enough to GR spacetime, is a physically real derivative entity. Finally, the derivative space view delivers, apparently, a solution to the problem of empirical coherence. Christian Wüthrich describes this point as follows:

[It] is a necessary condition for an empirical science that we can at least in principle measure or observe something at some location at some time. 
The italicized locution, in turn, seems to presuppose the existence of space and time. If that existence is now denied in quantum theories of gravity, one might then fear that these theories bid adieu to empirical science altogether. It thus becomes paramount for advocates of these theories to show that the latter only threaten the fundamentality, but not the existence of space and time. (Wüthrich 2017: 298)

In distinguishing between the fundamentality and the existence of spacetime, Wüthrich expresses the view that the two following claims are consistent: spacetime exists, and spacetime is not fundamental. The natural interpretation of this conjunction of claims is that spacetime has derivative existence, but lacks fundamental existence. Measurements and observations are occurring in (derivative) space and time.

Of course, if we solve the problem of empirical coherence by stipulating the existence of a derivative space, new issues follow from the fact that the natural world includes two related structures: the fundamental structure and the derivative space. One may ask to which category the connecting relation between the two structures is supposed to belong. A natural candidate, quite popular these days, is the relation of grounding (see for instance Fine 2001, Schaffer 2003, Correia and Schnieder 2012, and Wilson 2017). However, as others, I believe that the grounding relation should be understood as an explanatory relation, not a mind-independent relation obtaining in the world. ${ }^{19}$ Keeping in mind that the grounding relation will be understood here as an explanatory relation, let me use instead the notion of building relation, understood as an ontological mind-independent relation existing between entities. ${ }^{20}$ What matters is that the building relation, whatever it is, does not come for free in our ontology. If we do not need to posit a mind-independent relation, we should try to describe the world without using it. Furthermore, the derivative space view implies the existence of levels of reality: the ontological cost gets bigger and bigger. The notion of ontological level is not very clear, at least not as much as the notion of descriptive level. What does it mean that behind levels of description (think for instance of

${ }^{19}$ See Miller and Norton 2017. If you disagree, taking the grounding relation as a mind-independent relation, feel free to read 'grounding relation' where I write 'building relation'; nothing substantial follows from this terminological choice.

${ }^{20}$ See Bennett 2017, section 2. 
the biological level or the chemical level) lie 'ontological levels'? One could argue that levels come for free and should not be interpreted too seriously. However, if ontological levels come for free, then these merely are levels of description: the notion of ontological level has no counterpart obtaining in the world. The derivative view thereby collapses into eliminativism.

But perhaps one may argue that this is not a genuine problem. After all, maybe the ontological cost is well motivated insofar as it offers an adequate characterization of the delicate situation we face in contemporary physics. Nonetheless, if it is possible to come up with a view that does not entail the existence of levels of reality and has the same power of explanation, it should be preferred over the derivative space view. Or, at the very least, if you do not believe that ontological parsimony should be a too constraining criterion, the fact that space constitution is consistent with a reductionist ontology shows that the puzzlement triggered by the problem of space constitution is not in itself a reason favouring the existence of a derivative space, of layers of reality and of more-fundamental-than relations obtaining between entities inhabiting these levels.

We shall now turn to mereology in order to advance such an interpretation of space constitution, namely that space is a mereological sum of non-spatial entities. As we will see, the mereological view inherits some advantages of both eliminativism and the derivative view and avoids any commitment to levels of reality and to ontological priority.

\section{Mereology}

According to the mereological view of space constitution, the socalled 'derivative structure' is in fact a mereological sum of non-spatial building blocks. As such, space is not a genuinely derivative structure. Space is, in some non-spatial sense, within the fundamental structure. As a result, I suggest replacing the distinction between fundamental and derivative structures by a distinction between maximal and partial structures. Space is a partial structure, namely a proper part of the maximal structure. And there is no genuine distinction between fundamental and derivative entities (understanding fundamentality as ontological priority). Indeed, the mereological view acknowledges 
the reality of space, but it is also consistent with a reductionist ontology in which there are no levels of reality.

In order to defend that space is composed of non-spatial building blocks, it is useful to make a distinction between two claims. First, mereology can apply to space: space can be consistently approached as being a mereological sum of (at least) spatial relations. Indeed, the relationist Leibnizian approach may be expressed as the view that space is identical with a mereological sum of relations. The substantivalist view may be expressed as the view that space is identical to a substance, or a collection of substances. Nonetheless, if space is identical to one substance, or a collection of substances, it remains that the one substance is internally structured by spatial relations (in the monist framework), or that the various substances (in the pluralist framework) are structured by external spatial relations. Therefore, independently of whether space is only relational, or also substantial, it has to be identical to a collection of spatial relations (in the relationist picture), or to a collection of spatial relations and a 'substantial something' (in the substantivalist picture). What matters here is that the mereological sum associated with space will have to include at least spatial relations, independently of what the other ingredients will turn out to be: points, objects, properties, or substances (cf. Le Bihan 2016). Furthermore, these spatial relations may well instantiate properties of various kinds - in order to account for the curvature of space, for instance. Therefore, the view that space is identical to a collection of relations is consistent with the claim that space has a rich and complex structure. If the reader believes that it is awkward to describe space as being a mereological sum of spatial relations plus, possibly, other ingredients, what matters here is that the view is consistent. At the very least, it is logically consistent to conceive of any piece of space as being made of (i.e. composed of) spatial ingredients, and so, to conceive of the full physical space as being a mereological sum of geometrical building blocks.

Second, spatial building blocks of space (spatial relations) themselves can be made of non-spatial finer building blocks. Real difficulties start with this claim. If no spatial relations are to be found among the mereological atoms ${ }^{21}$ of space, how are we going to construe

\footnotetext{
${ }^{21}$ For the ease of presentation, I assume that the world has mereological at-
} 
the composition of spatial relations from entities that are not spatial? Although classical mereology (cf. Simons 1987) can easily apply to the composition of space, time or spacetime from spatial, temporal or spatio-temporal relations, it might be in tension with the composition of space, time or spacetime from entities that are not spatial, temporal or spatio-temporal. At least, classical mereology might have to be philosophically interpreted in an original way in order to build an approach wherein space can be construed as a mereological sum of non-spatial entities.

\section{The mereological view of space}

Could we simply assert that space is composed of non-spatial building blocks, or that spatial relations are collections of non-spatial entities? Denying that there is any problem with space composition from non-spatial building blocks would be one way to go, indeed. However, since there is something odd in the transition from the non-spatial to the spatial, one might argue that another explanatory move is required in order to make the claim plausible. For instance, if we focus on disordered locality in LQG, how could it be that a particular geometrical organization is made of a structure made of relations organized differently? In this section, I will assume for the sake of argumentation that there is prima facie something problematic with the identification of space constitution with space composition because of this strange explanatory gap between the sets of primitives notions involved in the two theories and suggest a way to avoid the problem. But note that if you believe that there is no genuine explanatory gap, it follows immediately that reductionism is a third, non-problematic way to interpret space constitution (see Le Bihan and Linnemann (forthcoming) for a study of the explanatory gap).

Logical mereology was developed as an approach of material objects (Paul 2002, 2006) in order to solve philosophical puzzles that I

oms. However, the mereological view is consistent with the claim that the world is gunky, i.e. that the world does not have mereological atoms. In this view, decomposition obtains all the way down, infinitely, and any proper part of the world has proper parts. For a discussion of the gunk view with respect to spacetime and the existence of objects, see e.g. Sider 1993, Le Bihan 2013 and Benovsky 2016. 
cannot describe here, and extended to the composition of space out of a physical configuration space in the context of quantum mechanics (2012). L.A. Paul argues in favour of a bundle theory of material objects, the view that material objects are only made of properties without substrates to instantiate or bear these properties. The properties are tied together by a bundling relation. According to L.A. Paul's mereological bundle theory, this bundling relation is identical to the relation of mereological composition. ${ }^{22}$ Ordinary objects are regarded as mereological sums of entities which do not belong themselves to the category of material objects but to the category of properties. Properties are parts, logical parts, of material objects. The term 'logical' should not confuse us, though. The relation of logical composition is mind-independent and concrete. It is mind-independent as it obtains independently of any observer and exists on its own. It is concrete as it has the same existential status as physical entities. The word 'logical' is here to express the idea that parts and wholes are not (only) carving reality at its geometrical joints, but at its categorical joints. An object is, allegedly, made of its spatial parts, but also, if we are ready to buy into L.A. Paul's notion of logical part, of its properties. Therefore, logical mereology allows trans-categorical composition, namely that entities belonging to a particular metaphysical category (say, properties) compose an entity that belongs to a distinct metaphysical category (say, an object or an event).

I will now present the mereological bundle theory of space, which avoids positing levels of reality. I will then describe more precisely how the view differs from L.A. Paul's view. According to the mereological bundle theory of space, the relation of constitution is identical to logical composition, and each of the constituted entities is a mereological bundle of proper parts of the maximal structure. In this framework, 3D spatial relations (in WFR) and 4D spatiotemporal relations (in general relativity) are interpreted as being logically made of parts belonging to distinct metaphysical categories.

${ }^{22}$ L.A. Paul conceives of the relation of composition as restricted (composition sometimes occurs, sometimes not), but this is not an essential component of the mereological bundle theory in general. Although the view is not defended in the literature, to my knowledge, one might endorse the view that material objects are generated by the unrestricted composition of primitive ingredients, entailing that any conceivable sum of properties actually is an object. 
The entity we call 'space' is a trans-categorical mereological sum of mereological atoms (following a bottom-up description) or, equivalently, a trans-categorical proper part of the maximal structure (following a top-down description). Importantly (in order to make sense of geometrical deviation in LQG), trans-categorical proper parts of a spatial relation do not have to be connected in the maximal structure. We can consider any distribution of entities, and ask whether this distribution is a mereological sum. And regarding whether or not a distributional class of entities composes a collective class, namely a mereological sum of these entities, the answer will be given by the actual derivation of the spatial description from the non-spatial theory. Composition occurs when, and only when, we may map an entity from the spatial structure onto a plurality of entities that are parts of the non-spatial structure. As a result, the mereological bundle theory of space entails a restricted composition answer to van Inwagen's special composition question (1990), which might count as a good or bad point, depending on one's philosophical view about the range of composition.

Operating under these assumptions, we end up with two structures, namely two collections of relations that permit us to localize entities. Some of the relations or other categories that compose the maximal structure, also compose trans-categorical sums - and these trans-categorical sums are spatial or spatio-temporal relations. Also, each connection between two constituted spatial or spatio-temporal relation is a logical mereological sum made of ingredients that are parts of the maximal structure. Therefore, trans-categorical composition plays two functions: first, composing each spatial or spatiotemporal relations; second, composing the whole spatial system of locality by also composing the connections between these relations, namely the organization of spatial or spatio-temporal relations.

Take the case of LQG and consider a fragment of GR spacetime: this fragment includes a constituted system of locality with relations of partial order between events or points. According to the mereological view, each of these relations of partial order is a mereological sum of entities localised, and potentially dispersed, in the maximal structure. But really, in the mereological framework, each of these relations of partial order is numerically identical to a mereological sum of logical proper parts of the maximal structure. These 
ingredients can be both scattered in the maximal structure by being non-local with respect to the system of locality associated with the maximal structure and local in the constituted spacetime, namely the partial structure we refer to as 'spacetime'. Each spatial or spatio-temporal relation that constitutes space (and looks like a primitive entity) is in fact made of trans-categorical parts. The mapping between the non-spatio-temporal building blocks and the spatial or spatio-temporal entities may well be very complex and strike us as weird, but there is no insurmountable difficulty here. Each relation that constitutes space is itself made of trans-categorical parts, and the whole space structure results from a relation of composition applied to each spatial relation.

Now, I will describe four ways in which my mereological approach differs from L.A. Paul's: a) composite entities are not necessarily objects; b) mereological simples are not necessarily properties; c) property instantiation is not parthood; d) parts are not more fundamental than the wholes that they compose.

(a) Composite entities are not necessarily objects. L.A. Paul conceived of her view as describing fusions of properties, which we identify with objects (both in the context of discussions about the nature of ordinary objects, and of discussions about the constitution of 3D objects from a $3 \mathrm{~N}$ space). Because of the focus on material objects (2002) and on WFR (2012), Paul focused on the composition of objects from mereological atoms. But note that there is no reason why a composed entity must belong to the category of material objects. Although material objects might well be bundles of mereological entities, in the context of the recovering of GR I propose to identify the composite entities with spatio-temporal relations. Note that there is some flexibility in the account regarding how we should think about the category of spatial entities. The category of spatial entities may have different categorical structures depending on whether space is analysed as a collection of relations only, or as a substance, for instance.

(b) Mereological simples are not necessarily properties. In the same way a chair is regarded as being made of various properties according to Paul (2002), space is here regarded as being made of various ingredients: $3 N$ relations, spin networks, or whatever else should be posited by physicists. These ingredients are not in space, and they are not 
space itself. They are building blocks of space. And maybe the building blocks belong to particular physical categories like spin networks, energy or $3 N$ relations. Perhaps we should categorize them as general metaphysical categories like properties or relations. Further work has to be done in order to determine to which categories the non-spatiotemporal mereological building blocks belong, and arguably, the result will differ in each particular approach to quantum gravity and WFR. But it should be noticed, in the case of quantum gravity, that the more the account is open to different categories at the mereologically fundamental level, the more it remains open to the success of several research programs in quantum gravity. ${ }^{23}$

In a way, and I think this is the spirit of L.A. Paul's account, which is supposed to be empirically flexible, namely open to the insight that we will get from physics, the account remains neutral on the categorical nature of the mereologically fundamental parts. It might be polyadic properties, but also points, relations which instantiates properties, substances or other categories. Although it is doubtful that physics will single out a unique set of categories as being mereologically fundamental, at least, these categories will have to be deployed in order to be as close as possible to the physical theory under consideration. ${ }^{24}$

${ }^{23}$ Also, it is very unlikely that the fundamental ontology will include 'primitive location properties' (properties of being at a particular coordinate) as proposed by Paul (2002) in order to solve the problem of material constitution. Indeed, if space is not a fundamental structure, it is hard to understand how location properties might be primitive ingredients fusing with other properties in order to compose spacetime.

${ }^{24}$ Importantly, in my picture quantum gravity has to describe the nature of mereologically fundamental entities, when analytic metaphysics, in association with effective theories like general relativity and quantum mechanics will have to account for the ontological categories involved in these structures. Here we must distinguish WFR and LQG (as an example of a QG program). If we focus on the best interpretation of quantum mechanics only, then the issue is about how to recover the description of a $3 \mathrm{D}$ world from a $3 \mathrm{~N}$ world, and the mereological bundle theory of $3 \mathrm{~N}$ relations will offer such a theory. Alternatively, if we focus on the broader picture including quantum gravity, it may well be that we need a three levels description, with the $3 N$ world being made of components from the ontology described by quantum gravity, and the $3 \mathrm{D}$ world being made of entities parts of the $3 N$ structure. 
(c) I also reject the claim that natural instantiation is parthood, namely that a natural object instantiates a natural property iff this property is one of its proper parts. For instance, one possible theory of instantiation that fits nicely with the account is that properties and relations are instantiated by the sole fact of being connected to other actual entities (cf. Le Bihan 2016).

(d) Finally, and most importantly, L.A. Paul endorses the ontological priority of the parts over the whole. ${ }^{25}$ Therefore, L.A. Paul's approach commits her to a stratified picture, giving ontological priority to the mereologically fundamental level. But this claim is independent of the mereological bundle theory of constituted entities (or material objects). One may accept the claim that constituted entities are mereological bundles of entities composing the maximal structure, and refuse the further claim that these parts are metaphysically prior to the wholes they logically compose. The view is compatible with the claim that wholes are more fundamental than parts and, interestingly for our purpose, that neither parts nor wholes are more fundamental than the other (in the sense of ontological priority). The mereological bundle theory, thereby, does not entail the existence of levels of reality since parts are not more fundamental than the wholes they compose. As such, there is no reason to believe that non-spatial parts belong to a more fundamental level, or that spatial wholes (spatial relations) belong to a more derivative level. Therefore, the mereological view avoids positing levels and allows us to stick to the more traditional view that the world is made of parts, the spatial and the non-spatial theories corresponding to particular 'mereological levels'.

The mereological theory of space thereby inherits the advantages of the derivative space view since it accepts the reality of space: there is no phenomenological issue since both our measurements and perceptions are occurring within time and space (or spacetime). The view avoids positing genuine derivative entities and allows us to adopt an ontology that does not include levels of reality. Another interesting advantage of the mereological view over the derivative

\footnotetext{
${ }^{25}$ See for instance Paul (2012: 221, footnote 1). Paul contrasts her view with the approach of Schaffer (2010) who takes wholes to be metaphysically prior to their parts.
} 
space view is that the relation of constitution is identified with a generic relation, namely one we find in many places in our ordinary and scientific descriptions of the world: the relation of composition. There is no need to posit a new and theoretically costly relation of emergence, designed specifically to do the work we expect it to do. One may object that this is true of the standard relation of composition, but not of the trans-categorical interpretation of composition. I agree: the latter might not be part of our naïve ontology. But it does not mean that the relation of trans-categorical composition does not explain a lot in many different contexts. I suggest that since the notion can do so much explanatory work (both in contemporary physics and in metaphysics, as established by L.A. Paul) without positing levels, it is an interesting option to take it as a primitive theoretical notion in our ontological interpretation of LQG and WFR.

Perhaps the reader will object to this line of thought that transcategorical composition is just another name for emergence (in the philosopher's sense), though: with the substitution of metaphysical emergence by trans-categorical composition, we have not accomplished much. However, there is no ontological priority of the parts over the whole, or of the wholes over their parts. The mereological approach states that the natural world is made of non-spatial parts, and that these parts are not more fundamental than the spatial relations they compose. To put it differently, we do not need to take seriously the claim that building blocks of space are literally building space. Building blocks are as much the result of a trans-categorical decomposition from the maximal structure, since there is no privileged ontological direction added to composition and decomposition. This is a crucial difference with respect to emergence as usually construed. Indeed, emergence is regarded as being asymmetric and generating ontological levels, in this context.

Finally, let me clarify briefly a methodological point. With composition we have a primitive theoretical notion that does a lot of explanatory work. The relation has a cost because, after all, we have to accept in the first place the existence of this relation if we want it to do the job. But, arguably, any metaphysical account of space constitution will have to rely on some primitive notion that explains the shift from the non-spatial to the spatial (leaving aside eliminativism). To put it differently, what we need is an interesting primitive notion acting 
as problem-solver in the context of space constitution (for an analysis of these two notions, see Benovsky 2010). The particular problemsolver that I have considered (composition) must be weighted in comparison with the generic relation of constitution that we find in contemporary physics ('emergence') and with other building relations that could be used to analyse the neutral relation of constitution. What matters here is that composition is a particular building relation, a particular problem-solver, that clearly avoids an ontologically loaded interpretation of the levels involved in space constitution, and avoids positing a relation of ontological priority obtaining between the levels. To be crystal clear: it does not show that space constitution should necessarily be interpreted in a mereological framework. But the very existence of this levels-free problem-solver shows that space constitution does not entail the existence of ontological levels connected by relations of ontological priority.

\section{Conclusion}

The mereological view of space offers an interesting middle way between eliminativism and the derivative space view. Space is a mereological bundle of non-spatial building blocks. It is neither derivatively real nor fundamentally real since there are no levels of reality and no separation between fundamental and derivative entities. The view inherits many advantages of the derivative space view: it solves the problem of empirical coherence and the phenomenal issue by accepting the reality of space. But it does so at a lesser cost by avoiding committing to a stratified ontology. Thus, space constitution is not necessarily pointing towards the existence of ontological levels within physics, and we should not be too quick to see there a new motivation for adopting anti-reductionism about special sciences. More generally, composition is an excellent primitive theoretical notion to interpret space constitution, without positing unnecessary entities (levels and non-fundamental entities). Therefore, the phenomenon of space constitution, if it had to be confirmed in one of the research programs in quantum gravity or if WFR turned out to be the best reading of quantum mechanics, would not commit us to a strong relation of emergence: with trans-categorical mereology, we can get rid of emergence (in 
the philosopher's sense), fundamentality as ontological priority and levels of reality. ${ }^{26}$

Baptiste Le Bihan

Department of Philosophy

University of Geneva

2 rue de Candolle

Geneva

Baptiste.LeBihan@unige.ch

\section{References}

Albert, David Z. 2015. After Physics. Harvard University Press.

Bennett, Karen. 2017. Making Things Up. Oxford: Oxford University Press.

Benovsky, Jiri. 2010. Relational and substantival ontologies, and the nature and the role of primitives in ontological theories. Erkenntnis 73(1): 101-121.

Benovsky, Jiri. 2015. From experience to metaphysics: on experience-based intuitions and their role in metaphysics. Noûs 49(4): 684-97.

Benovsky, Jiri. 2016. Eliminativism and gunk. Teorema: International Journal of Philosophy 35(1): 59-66.

Butterfield, Jeremy. 2011. Emergence, reduction and supervenience: a varied landscape. Foundations of Physics 41(6): 920-59.

Chalmers, David J. 1995. Facing up to the problem of consciousness. Journal of Consciousness Studies 2(3): 200-19.

Chalmers, David J. Forthcoming. The meta-problem of consciousness, manuscript, https://philpapers.org/archive/CHATMO-32.pdf.

Correia, Fabrice; and Schnieder, Benjamin 2012. Grounding: An Opinionated Introduction. In Metaphysical Grounding: Understanding the Structure of Reality, edited by F. Correia and B. Schnieder. Cambridge University Press.

Crowther, Karen J. 2016. Effective Spacetime: Understanding Emergence in Effective Field Theory and Quantum Gravity. New York, NY: Springer.

${ }^{26}$ I wish to thank Augustin Baas, Jiri Benovsky, Claudio Calosi, Lorenzo Cocco, Fabrice Correia, Karen Crowther, Michael Esfeld, Nick Huggett, Rasmus Jaksland, Niels Linnemann, Vincent Lam, Keizo Matsubara, Sebastian Murgueitio Ramirez, Christian Wüthrich, David Yates, an anonymous referee and the audiences at the workshop Metaphysics and Physics: Methodological Links (University of Lausanne) and at the Third Annual Conference of the Society for the Metaphysics of Science (Fordham University) for valuable feedback. This work was performed under a collaborative agreement between the University of Illinois at Chicago and the University of Geneva and made possible by grant number 56314 from the John Templeton Foundation. Its contents are solely the responsibility of the author and do not necessarily represent the official views of the John Templeton Foundation. 
Crowther, Karen J. 2018. Inter-theory relations in quantum gravity: correspondence, reduction, and emergence. Studies in History and Philosophy of Science Part B: Studies in History and Philosophy of Modern Physics 63: 74-85.

Fine, Kit. 2001. The question of realism. Philosophers' Imprint 1(1): 1-30.

Heil, John. 2003a. From an Ontological Point of View. Oxford University Press. Heil, John. 2003b. Levels of reality. Ratio 16(3): 205-21.

Huggett, Nick. 2017. Target space $\neq$ space. Studies in History and Philosophy of Science Part B: Studies in History and Philosophy of Modern Physics. doi:10.1016/j. shpsb.2015.08.007.

Huggett, Nick; and Wüthrich, Christian. 2013. Emergent spacetime and empirical (in)coherence. Studies in History and Philosophy of Science Part B: Studies in History and Philosophy of Modern Physics 44(3): 276-85.

Humphreys, Paul. 2016. Emergence. New York: Oxford University Press.

Lam, Vincent; and Wüthrich, Christian. Forthcoming. Spacetime is as spacetime does. Studies in History and Philosophy of Science Part B: Studies in History and Philosophy of Modern Physics 64: 39-51.

Le Bihan, Baptiste. 2013. Why a gunk world is compatible with nihilism about objects. Studia Philosophica Estonica 6(1): 1-14.

Le Bihan, Baptiste. 2015. The unrealities of time. Dialogue 54(1): 25-44.

Le Bihan, Baptiste. 2016. Super-relationism: combining eliminativism about objects and relationism about spacetime. Philosophical Studies 173(8): 2151-72.

Le Bihan, Baptiste. 2018. Priority monism beyond spacetime. Metaphysica, 19(1): 95-111.

Le Bihan, Baptiste; and Linnemann, Niels. Forthcoming. Have we lost spacetime on the way? Narrowing the gap between general relativity and quantum gravity. Studies in History and Philosophy of Science Part B: Studies in History and Philosophy of Modern Physics.

Le Bihan, Baptiste; and Read, James. Forthcoming. Duality and ontology. Philosophy Compass.

Lewis, Peter J. 2004. Life in configuration Space. British Journal for the Philosophy of Science 55(4): 713-29.

McLaughlin, Brian P. 1992. The rise and fall of British emergentism. In Emergence or Reduction? Prospects for Nonreductive Physicalism, edited by A. Beckermann, H. Flohr and J. Kim. Berlin: De Gruyter.

McTaggart, John. 1908. The unreality of time. Mind 17(68): 457-74.

Mellor, D. H. 1998. Real Time II. New York: Routledge.

Miller, Kristie; and James Norton. 2017. Grounding, it's (probably) all in the head. Philosophical Studies 174(12): 3059-81.

Monton, Bradley. 2002. Wave function ontology. Synthese 130(2): 265-77.

Monton, Bradley. 2006. Quantum mechanics and 3N dimensional space. Philosophy of Science 73(5): 778-89.

Ney, Alyssa. 2012. The status of our ordinary three dimensions in a quantum universe. Noûs 46(3): 525-60.

Ney, Alyssa. 2015. Fundamental physical ontologies and the constraint of 
empirical coherence: a defense of wave function realism. Synthese 192(10): $3105-24$.

Oaklander, L. Nathan. 1987. Temporal relations and temporal becoming: a defense of a Russellian theory of time. Noûs 21(1): 75-7.

Paul, L. A. 2002. Logical parts. Noûs 36(4): 578-96.

Paul, L. A. 2010. Temporal experience. Journal of Philosophy 107(7): 333-59.

Paul, L. A. 2012. Building the world from its fundamental constituents. Philosophical Studies 158(2): 221-56.

Rovelli, Carlo. 2004. Quantum Gravity. Cambridge, Cambridge University Press. Rovelli, Carlo; and Vidotto, Francesca. 2014. Covariant Loop Quantum Gravity. Cambridge: Cambridge University Press.

Schaffer, Jonathan. 2003. Is there a fundamental level? Noûs 37(3): 498-517.

Schaffer, Jonathan. 2010. Monism: the priority of the whole. Philosophical Review 119(1): 31-76.

Sider, Theodore. 1993. Van Inwagen and the possibility of gunk. Analysis 53(4): 285-9.

Simons, Peter M. 1987. Parts: A Study in Ontology. Oxford University Press.

Van Inwagen, Peter. 1990. Material Beings. Ithaca: Cornell University Press.

Wilson, Alastair. 2017. Metaphysical causation. Noûs 50(4): 723-51.

Wüthrich, Christian. 2017. Raiders of the lost spacetime. In Towards a Theory of Spacetime Theories, edited by D. Lehmkuhl, G. Schiemann and E. Scholz. New York: Springer.

Yates, David. Forthcoming. Thinking about spacetime. In Philosophy Beyond Spacetime, edited by C. Wüthrich, B. Le Bihan and N. Huggett. Oxford: Oxford University Press. 